\title{
Thank you to our reviewers 2016
}

The Editors would like to publicly acknowledge the people listed below who served as reviewers on the journal during 2016. Without their efforts, the quality of the journal could not be sustained.

$\begin{array}{ll}\text { Abib, Fernando C } & \text { Hsuan, James } \\ \text { Ahmad, Sajjad } & \text { Hu, Victor } \\ \text { Antunes, Victor } & \text { Jayaram, Hari } \\ \text { Arden, G B } & \text { Jiao, Shuliang } \\ \text { Balikoglu-Yilmaz, Melike } & \text { Karanth, Swathi } \\ \text { Bansal, Rakesh Kumar } & \text { Kasturi, Nirupama } \\ \text { Baylis, Oliver } & \text { Kelly, Simon } \\ \text { Bhargava, Rahul } & \text { Kim, Jee Taek } \\ \text { Bui, Bang } & \text { King, Anthony J } \\ \text { Cañadas Suarez, Pilar } & \text { Kitazawa, Koji } \\ \text { Chakrabati, Arup } & \text { Knier, Benjamin } \\ \text { Chan, Tommy C Y } & \text { Kowalski, Regis P } \\ \text { Cheeseman, Robert } & \text { Kutty, Shankar Ayyappan } \\ \text { Chen, Haoyu } & \text { Lansingh, Van Charles } \\ \text { Costagliola, Ciro } & \text { Lapid-Gortzak, Ruth } \\ \text { Czanner, Gabriela } & \text { Larin, K V } \\ \text { Dey, Pranab } & \text { Lascaratos, Gerassimos } \\ \text { Di Zazzo, Antonio } & \text { Lee, Jacky W Y } \\ \text { Evans, Jennifer R } & \text { Lee, Junyeop } \\ \text { Fialová, Stanislava } & \text { Li, Bing } \\ \text { Frisher, Martin } & \text { Limburg, Hans } \\ \text { Ghoneim, Ehab } & \text { Liu, Quan } \\ \text { Giansanti, Fabrizio } & \text { MacDonald, Susan } \\ \text { Groh, Janos } & \text { MacIntyre, Ross }\end{array}$

Harris, William

\author{
Mbadugha, Chigozie \\ Anuli \\ McGhee, Charles N J \\ Milazzo, S \\ Mohammed, Abdull \\ Morrison, John \\ MR, Sriram Gopal \\ Munemasa, Yasunari \\ Murakami, Tomoaki \\ Murlimanju, B V \\ Nanavaty, Mayank A \\ Neal, Tim \\ O'Neill, Evelyn \\ Oner, Veysi \\ Ostri, Christoffer \\ Panda, Anita \\ Piagkou, Maria \\ Pinna, Antonio \\ Ranjan, Pratyush \\ Reddy, Sagili \\ Chandrasekhara \\ Redfern, William S \\ Rewri, Parveen \\ Romano, Mario \\ Romano, Vito
}

Schargus, Marc

Seidel, Gerald

Shih, Yen-Yu I

Sinawat, $\mathrm{S}$

Singh, Sumit

Smyth, Bobby

Stappler, Theodor

Steel, David

Stewart, Rosalind

Stojanovic, Aleksandar

Sugar, Joel

Tabin, Geoff

Todd, D A

Tosini, Gianluca

Urban, Beata

Vande Geest, Jonathan P

Vardi, Noga

Viola, Francesco

Viso, Eloy

Wang, Yan

Wiggs, Janey L

Yang, Lin

Zhao, Yitian

Zheng, Yalin

Zhou, Xingtao 